\title{
320 - Barriers to Deprescribing in Older Adults with Dementia
}

Mariana Duarte-Mangas; Gonçalo A. Santos; Beatriz Jorge; Catarina Pedro Fernandes

Individuals with dementia usually have multiple chronic illnesses, most of whom are medicated with five or more medications. However, as dementia progresses and the goal of care moves from prolonging life to optimizing quality of life, the risk of taking certain medications may outweigh its benefit. Therefore, it is necessary to reassess the medication over time.

The objective of this work was to explore the barriers to optimising prescribing and deprescribing (withdrawing) of medications and the benefits of this procedure. Optimizing pharmacological treatment for people with dementia usually requires the prescription of inappropriate drugs and the initiation of other drugs. Several obstacles to the optimization of treatment have been identified in elderly people with multiple morbidities, related to the doctor, the health system, the patient and the caregiver, including: inadequate guidelines, incomplete medical history, prevention of negative consequences and established beliefs in the benefits and harms medication. Desprescribing older people with dementia is made even more difficult by the decrease in decision-making capacity, difficulties in understanding and communicating, increased involvement of caregivers and difficulties in setting goals. 